\title{
Covid 19 and gastrointestinal symptoms: A case report of a Mesenteric Large vessel obstruction
}

\author{
souhir nasseh ${ }^{1}$, Med Mehdi Trabelsi ${ }^{1}$, Nabil Haloui $^{1}$, annouar oueslati ${ }^{1}$, hichem jerraya ${ }^{2}$, \\ and Ramzi Nouira ${ }^{2}$ \\ ${ }^{1}$ Charles Nicolle Hospital \\ ${ }^{2}$ Faculty of medecine of tunis
}

February 26, 2021

\begin{abstract}
COVID-19 cases are increasing worldwide. With such emerging disease the medical community should be aware of atypical clinical presentations in order to help with correct diagnosis, and to take the proper measures to isolate and treat patients to avoid healthcare professionals being infected and to limit its spread (SARS-CoV-2).
\end{abstract}

\section{Introduction}

COVID-19 cases are increasing daily worldwide. With such emerging disease the medical community should be aware of atypical clinical presentations in order to help with correct diagnosis, and to take the proper measures to isolate and treat patients to avoid healthcare professionals being infected and to limit its spread (SARS-CoV-2).

The typical clinical manifestations are general and respiratory symptoms. Less common symptoms included headache, diarrhea, nausea and vomiting. However, diarrhea and abdominal pain as the first symptom is rarely reported.

The pathophysiology of these two symptoms likely relies on an inflammatory process such as gastroenteritis among other reasons

$[1]$.

Parry et al recognized COVID-19-associated coagulopathy as one of the key drivers of mesenteric vascular thrombosis; however, they mentioned there was lack of both radiologic and histologic evidence for large arterial and venous mesenteric vessel thrombosis [2].

We report a case of covid 19 causing mesenteric ischemia diagnosed early and has been treated with Heparin.

\section{Case report}

A 68-year-old no smoking male patient with no past medical history, presented with epigastric pain and diarrhea since 4 days. In the physical examination he was febrile at $38.5^{\circ} \mathrm{C}$ and he has hypogastric tenderness. Blood test showed WBC $17660 / \mathrm{mL}$ and C-reactive protein 125mg/l. A CT scan showed a thickening of the right colon wall and the last $30 \mathrm{~cm}$ of the small bowl associated (Fig 2-3) with an obstruction of the terminal segment of the ilio colic artery (Fig1). There were no other signs of ischemia. We decided to put the patient under medical treatment with unfractionned heparine. On the second day of his stay he developed a dry cough and the abdominal pain decreased. We performed a laparoscopy on the third day that showed a normal condition of the colon and the small bowl. With the appearance of respiratory signs and the pandemic 
context, we decided to perform a COVID-19 RT-PCR test to the patient. He tested positive. After 1 week of treatment, the patient is asymptomatic with soft abdomen and normal rate of WBC and C-reactive protein.

\section{Discussion}

This case of mesenteric ischemia in a patient with COVID 19, with no risk of thrombogenesis highlights the variability of symptoms of this disease.

To our knowledge, we are among the first who described a case of mesenteric large vessel obstruction associated to the Corona virus worldwide.

CT scan is known to be one of diagnostic means of COVID-19. Besides bilateral multiple ground glass opacities at lung bases in chest CT scan, abdominal imaging findings such as small and large bowel wall thickening, fluid-filled colon, pneumatosis intestinalis, pneumoperitoneum, intussusception and ascites have also been reported[3] .

Thrombogenesis in COVID-19 has also been described in few cases and it can be attributed to the direct and indirect cytotoxic effects of the SARS-CoV-2 virus on vascular endothelium, due to the corona virus's affinity to the ACE-2 receptor on endothelial surfaces. In fact, endothelial dysfunction is a principal determinant of micro vascular dysfunction by shifting the vascular equilibrium towards more vasoconstriction with subsequent organ ischemia, inflammation with associated tissue edema, and a pro coagulant state [4].

In our case, this phenomenon touched a large vessel rather than capillaries, which is why it was treatable by Heparine.

To reduce the contamination rate properly, it is imperative to recognize SARS-CoV-2-positive patients rapidly in order to deliver adapted care and to isolate them from the rest of the patients until their recovery. Early suspicion is also necessary to protect healthcare workers, who should use personal protective equipment in such cases.

It implies that, as long as the COVID-19 pandemic is ongoing, patients presenting with non-explained gastrointestinal symptoms should be managed as potentially infected and COVID-19 diagnostic tests should be performed in time such is our case.

\section{Conclusion}

Life threatening manifestations of the corona virus disease are related mostly to the respiratory system, but due to thrombogenesis, other manifestations in gastrointestinal tract should be taken into account such as acute mesenteric ischemia.

\section{References:}

1. Gérald Gahide,A Julien FrandonB and Jean-François Vendrell. COVID-19 patients presenting with afebrile acute abdominal pain. Royal College of Physicians 2020

2. Parry AH, Wani AH, Yaseen M. Acute mesentericischemia in severe coronavirus-19 (COVID-19): possible mechanisms and diagnostic pathway.AcadRadiol2020; 27:1190.

3. Abdominal Radiologyhttps://doi.org/10.1007/s00261-020-02739-5

4. Bonetti PO, Lerman LO, Lerman A. Endothelialdysfunction - a marker of atheroscleroticrisk.ArteriosclThrom Vas 2003; 23: 168-75. 


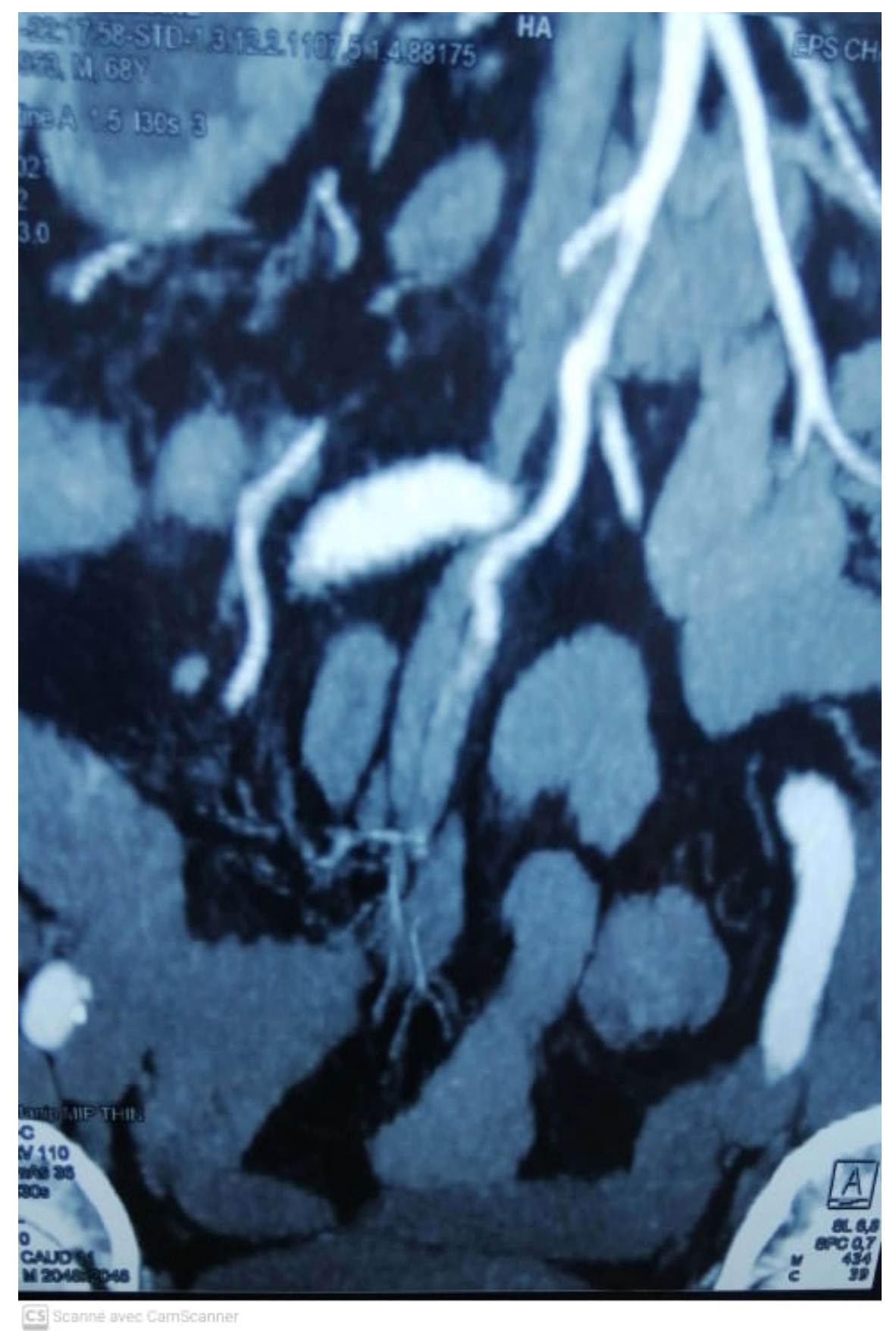



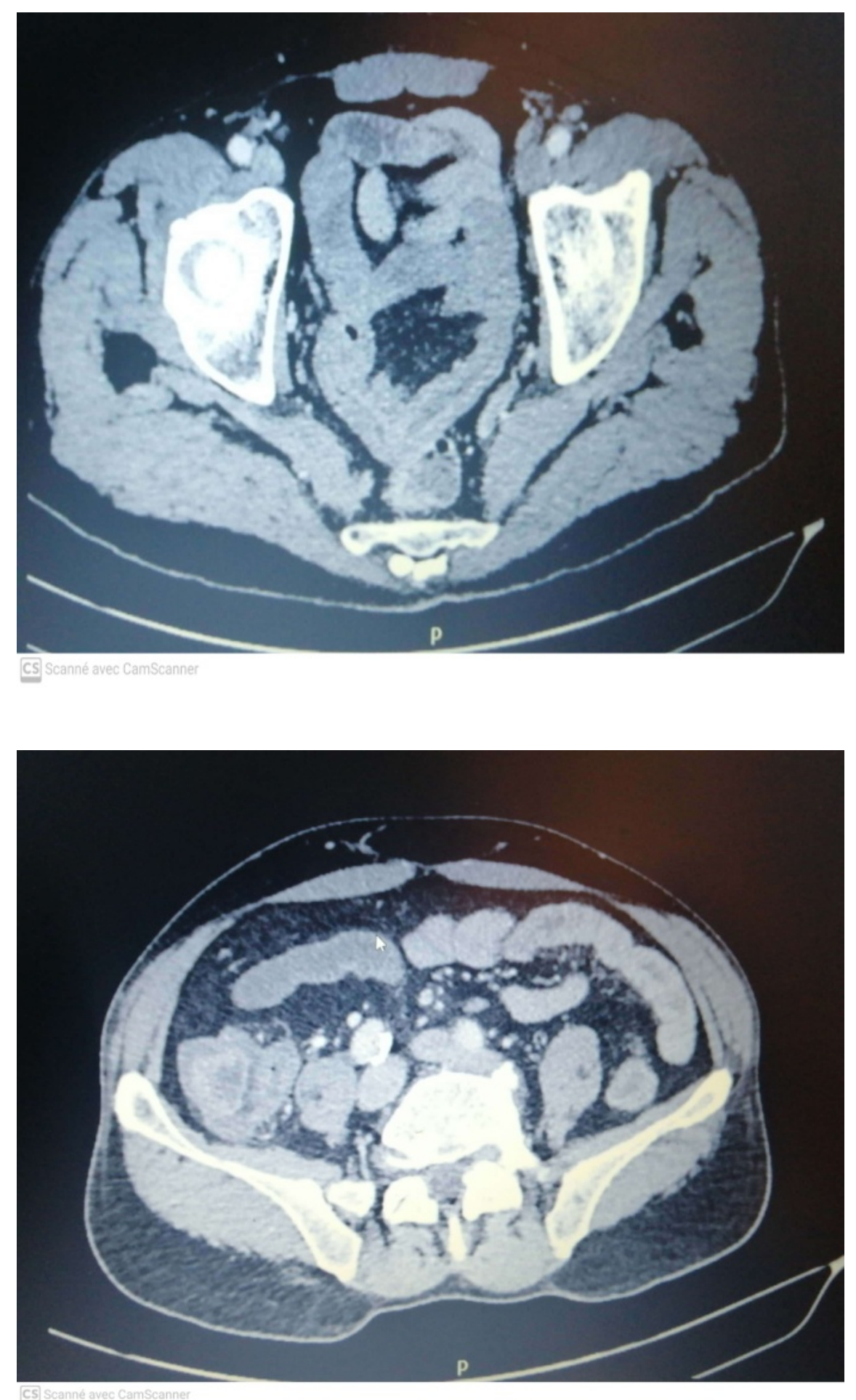


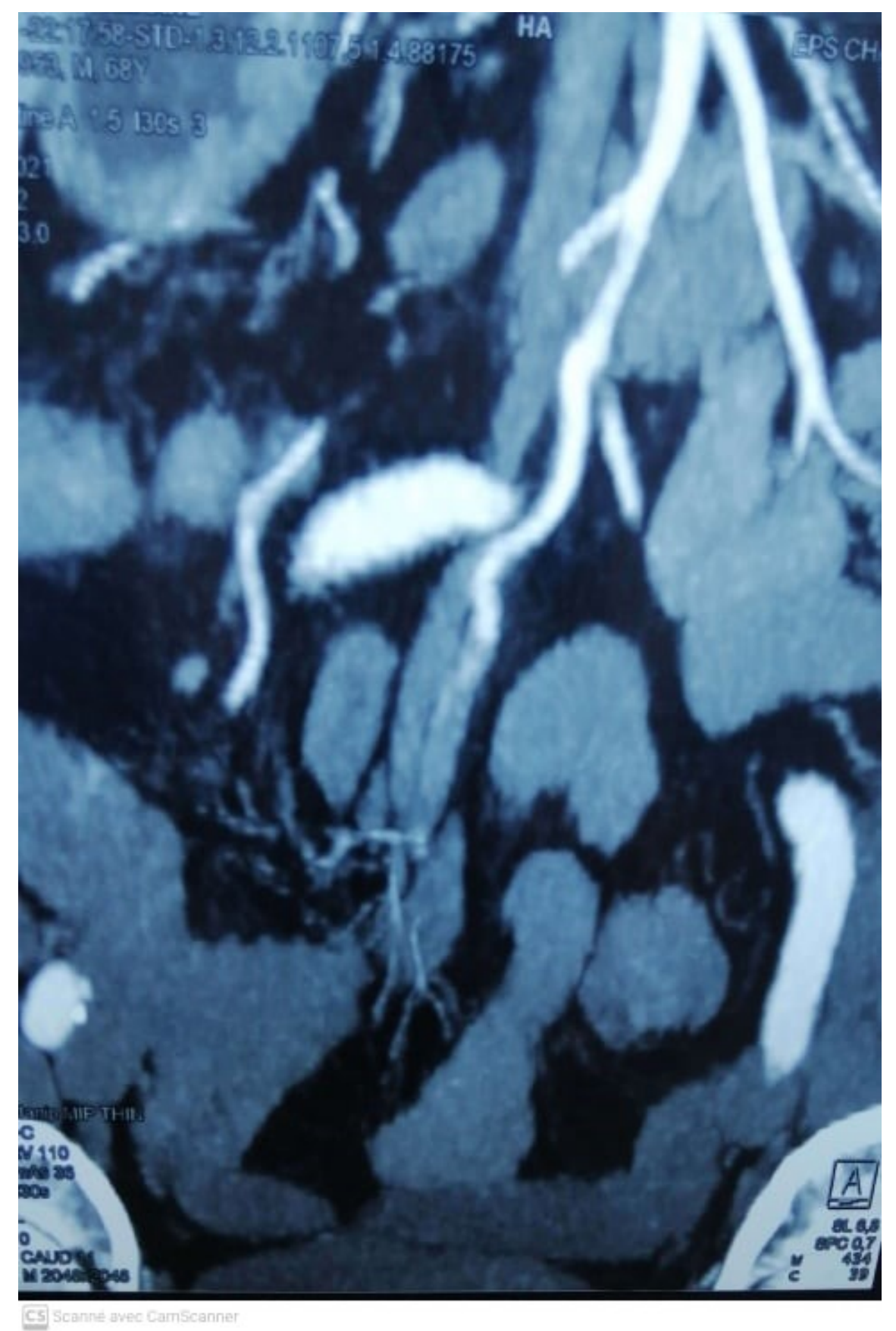



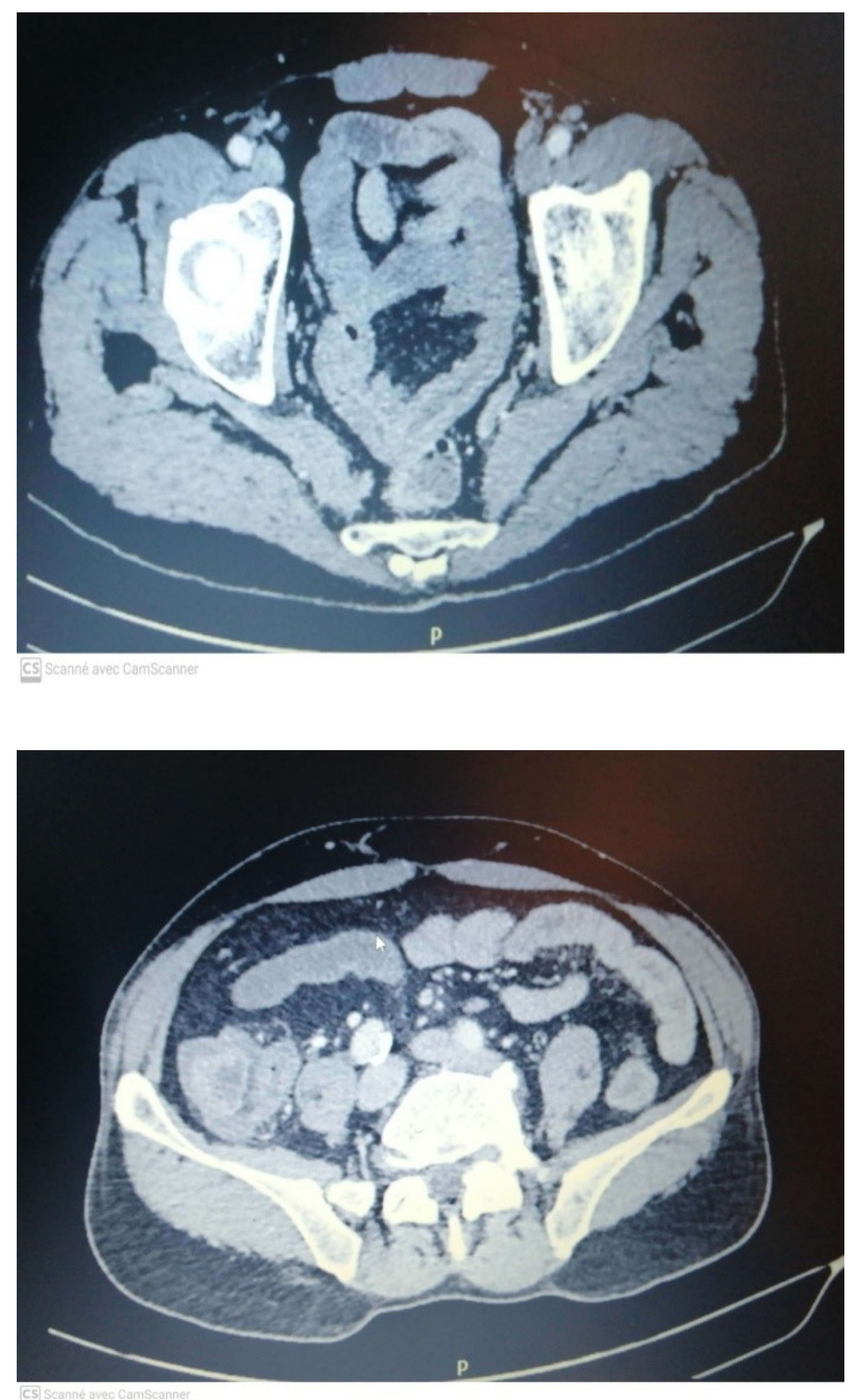